\title{
ON THE TOPOLOGICAL COMPLETION OF $M$-SPACE PRODUCTS
}

\author{
A. K. STEINER
}

Abstract. Using the continuum hypothesis, an example is given of two countably compact spaces $X$ and $Y$ such that $X \times Y$ is an $M$-space, but is not countably compact. If $\mu X$ denotes the completion of $X$ with respect to its finest uniformity, the above example shows that $\mu(X \times Y)$ is not necessarily equal to $\mu X \times \mu Y$, even though $X \times Y$ is an $M$-space.

It is known that the product of countably compact spaces need not be countably compact [7], and that the product of $M$-spaces need not be an $M$-space [4]. Since countably compact spaces are $M$-spaces, the question remains:

Q: If $X$ and $Y$ are countably compact and $X \times Y$ is an $M$-space, is $X \times Y$ countably compact?

In [5], [6], Morita defines $\mu X$ to be the topological completion of a completely regular $T_{1}$-space $X$ with respect to its finest uniformity and proves that if $X \times Y$ is an $M$-space, then $\mu(X \times Y)=\mu X \times \mu Y$ if and only if $K \times L$ is countably compact whenever $K$ and $L$ are closed countably compact subsets of $X$ and $Y$, respectively. In general, $\mu(X \times Y) \neq \mu X \times \mu Y$; however, in [6], Morita asks whether $\mu(X \times Y)$ $=\mu X \times \mu Y$ holds if $X \times Y$ is an $M$-space. He shows that this is equivalent to $Q$.

Assuming the continuum hypothesis, spaces $X$ and $Y$ are constructed to provided a negative answer to $Q$.

Throughout, all spaces are assumed to be completely regular and $T_{1}$; the word countable will mean countably infinite; $N$ is the set of positive integers with the discrete topology.

A space $K$ is countably compact if and only if each countable discrete subset of $K$ has an accumulation point. A space $X$ is an $M$-space if and only if it admits a closed, continuous mapping into a metric space such that point inverses are countably compact.

Since the spaces to be constructed are subsets of $\beta N$, the following facts will be reviewed.

If $M$ is a countable discrete subset of $\beta N$, the Stone-Cech compactification of $N$, then cl $M$ (the closure of $M$ in $\beta N$ ) is homeomorphic to $\beta N$, and $M^{*}=\operatorname{cl} M-M$ is homeomorphic to $N^{*}=\beta N-N$.

Received by the editors October 14, 1970.

AMS 1970 subject classifications. Primary 54G20; Secondary 54D20, 54C10.

$K e y$ words and phrases. Countable compactness, $M$-spaces. 
If $M_{1}$ and $M_{2}$ are disjoint countable subsets of $\beta N$ such that $M_{1} \cup M_{2}$ is discrete, then $\mathrm{cl} M_{1} \cap \mathrm{cl} M_{2}=\varnothing$ and $M_{1}^{*} \cap M_{2}^{*}=\varnothing$.

If $M_{1}$ and $M_{2}$ are countable discrete subsets of $\beta N, x \in M_{1}^{*}$ and $y \in M_{2}^{*}$, then the type of $x$ relative to $M_{1}$ is said to be the same as the type of $y$ relative to $M_{2}$ (and written $\tau\left(x, M_{1}\right)=\tau\left(y, M_{2}\right)$ ) if there is a homeomorphism of $\mathrm{cl} M_{1}$ on to $\mathrm{cl} M_{2}$ taking $x$ onto $y$.

A point $x \in M^{*}$ is a $P$-point in $M^{*}$ if every $G_{\delta}$ in $M^{*}$ is a neighborhood of $x$ in $M^{*}$. Assuming the continuum hypothesis, W. Rudin [8] showed that there are $2^{c} P$-points in $N^{*}$ (and hence in $M^{*}$ for any countable discrete $M \subset \beta N$ ).

Define an equivalence relation $\sim$ on the $P$-points in $M^{*}$ by $x \sim y$ if and only if $\tau(x, M)=\tau(y, M)$. Since there are only $c$ autohomeomorphisms on $\mathrm{cl} M$, there are $2^{c}$ equivalence classes of $P$-points.

LEMMA 1 (FRoLík [2]). If $M_{1}$ is a countable discrete subset of $\beta N$ and $M_{2} \subset M_{1}$, then $\tau\left(x, M_{2}\right)=\tau\left(x, M_{1}\right)$ for each $x \in M_{2}^{*}$.

LEMMA 2 (FROLÍk [3]). If $M_{1}$ and $M_{2}$ are countable discrete subsets of $\beta N$, then $M_{3}=\left(M_{1} \cap M_{2}\right) \cup\left(M_{1}^{*} \cap M_{2}\right) \cup\left(M_{1} \cap M_{2}^{*}\right)$ is discrete, $\operatorname{cl} M_{3}=\operatorname{cl} M_{1} \cap \mathrm{cl} M_{2}$, and $M_{3}^{*}=M_{1}^{*} \cap M_{2}^{*}$.

THEOREM. If $M_{1}$ and $M_{2}$ are countable discrete subsets of $\beta N$ and $x$ is a P-point in both $M_{1}^{*}$ and $M_{2}^{*}$, then $x \in\left(M_{1} \cap M_{2}\right)^{*}$ and $\tau\left(x, M_{1}\right)$ $=\tau\left(x, M_{2}\right)$.

Proof. Since $x \in M_{1}^{*} \cap M_{2}^{*}$, either $x \in\left(M_{1} \cap M_{2}\right)^{*}, x \in\left(M_{1}^{*} \cap M_{2}\right)^{*}$, or $x \in\left(M_{1} \cap M_{2}^{*}\right)^{*}$, by Lemma 2 . However, $x$ is a $P$-point in $M_{1}^{*}$ and so cannot be a limit point of any countable subset of $M_{1}^{*}$. Thus $x$ $\bigoplus\left(M_{1}^{*} \cap M_{2}\right)^{*}$. Similarly, $x \notin\left(M_{1} \cap M_{2}^{*}\right)^{*}$. Since $x \in\left(M_{1} \cap M_{2}\right)^{*}$ and $M_{1} \cap M_{2} \subset M_{i}$, for $i=1,2$, Lemma 1 implies that $\tau\left(x, M_{1}\right)$ $=\tau\left(x, M_{1} \cap M_{2}\right)=\tau\left(x, M_{2}\right)$.

Construction of $X$ and $Y$. The following example is a modification of the example of Novák [7], simplified by Frolík [1], of two countably compact spaces whose product is not countably compact.

$\beta N$ has $2^{c}$ countable discrete subsets which may be well ordered as $M_{1}, M_{2}, \cdots, M_{\alpha}, \cdots ; \alpha<\aleph$, where $\aleph$ is the smallest ordinal of cardinality $2^{c}$.

Let $\phi$ be a mapping from the countable discrete subsets of $\beta N$ into $N^{*}$, defined as follows:

$\phi\left[M_{1}\right]$ is a $P$-point in $M_{1}^{*}$. For each $\alpha, 1<\alpha<\aleph, \phi\left[M_{\alpha}\right]$ is a $P$-point in $M_{\alpha}^{*}$ such that $\tau\left(\phi\left[M_{\alpha}\right], M_{\alpha}\right) \neq \tau\left(\phi\left[M_{\beta}\right], M_{\beta}\right)$ for any $\beta<\alpha$. This is possible since there are $2^{c}$ equivalence classes of $P$-points in $M_{\alpha}^{*}$ and $\alpha$ has fewer than $2^{c}$ predecessors. 
Inductively, define a sequence $X_{0}, X_{1}, \cdots, X_{\gamma}, \cdots ; \gamma<\omega_{1}$ of subsets of $\beta N$ such that $X_{0}=N$ and for each $\gamma<\omega_{1}, X_{\gamma}$ $=\bigcup_{\alpha<\gamma} X_{\alpha} \cup\left\{\phi[M]: M\right.$ is a countable discrete $\left.\subset \cup_{\alpha<\gamma} X_{\alpha}\right\}$. Let $X=\bigcup\left\{X_{\gamma}: \gamma<\omega_{1}\right\}$. Since each countable discrete subset of $X$ is contained in $X_{\gamma}$ for some $\gamma<\omega_{1}$, it has an accumulation point in $X_{\gamma+1} \subset X$. $X$ is therefore countably compact. For each $\gamma<\omega_{1}$, card $X_{\gamma}$ $\leqq c \cdot c+(c \cdot c)^{\aleph_{0}}=c$, so card $X \leqq c$.

Let $Y=N \cup(\beta N-X)$. For each countable discrete $A \subset Y$, card cl $A=2^{c}$, so $A^{*} \cap Y \neq \varnothing$ and $A$ has an accumulation point in $Y$. Thus $Y$ is also countably compact.

$X \times Y$ is not countably compact. If $\Delta=\{(n, n): n \in N\}$, then $\Delta$ is a countable discrete subset of $X \times Y$. Any accumulation point of $\Delta$ must be of the form $(p, p)$ for $p \in N^{*}$. Since $X \cap Y=N$, the set $\Delta$ has no accumulation point in $X \times Y$.

$X \times Y-\Delta$ is countably compact. Let $\left\{\left(x_{i}, y_{i}\right)\right\}$ be a countable discrete subset of $X \times Y-\Delta$. If $x_{i}=x$ (or $y_{i}=y$ ) for an infinite number of indices, then $\left\{\left(x_{i}, y_{i}\right): x_{i}=x\right\}$ is a countable discrete subset of $\{x\}$ $X Y$, which is countably compact. $\left\{\left(x_{i}, y_{i}\right): x_{i}=x\right\}$ thus has an accumulation point in $\{x\} \times Y \subset X \times Y$. It may, then, be assumed that $x_{i} \neq x_{j}$ and $y_{i} \neq y_{j}$ if $i \neq j$. It may also be assumed (by choosing an appropriate subset) that $M_{1}=\left\{x_{i}\right\}$ and $M_{2}=\left\{y_{i}\right\}$ are discrete subsets of $X$ and $Y$, respectively, and that $M_{1} \cap M_{2}=\varnothing$ (since $x_{i} \neq y_{i}$ for all $i=1,2, \cdots)$.

Let $h$ be the homeomorphism of cl $M_{1}$ onto cl $M_{2}$ defined by $h\left(x_{i}\right)$ $=y_{i}$ for all $x_{i} \in M_{1}$. The point $\phi\left[M_{1}\right]$ is in $X$ and is in cl $M_{1}$. Since $h$ is a homeomorphism, $h \phi\left[M_{1}\right] \in \mathrm{cl} M_{2}, \tau\left(\phi\left[M_{1}\right], M_{1}\right)=\tau\left(h \phi\left[M_{1}\right], M_{2}\right)$ and $h \phi\left[M_{1}\right]$ is a $P$-point in $M_{2}^{*}$.

If $h \phi\left[M_{1}\right] \in X$, then $h \phi\left[M_{1}\right]=\phi[M]$ for some countable discrete $M \subset X$; that is, $h \phi\left[M_{1}\right]$ is a $P$-point in $M^{*}$. Since $h \phi\left[M_{1}\right]$ is a $P$-point in both $M^{*}$ and $M_{2}^{*}$, it follows from the theorem that $h \phi\left[M_{1}\right]$ $\in\left(M \cap M_{2}\right) *$ and $\tau\left(h \phi\left[M_{1}\right], M\right)=\tau\left(h \phi\left[M_{1}\right], M_{2}\right)$.

By the definition of $\phi$, the second relation gives $M=M_{1}$ (because $\left.\tau\left(h \phi\left[M_{1}\right], M_{2}\right)=\tau\left(\phi\left[M_{1}\right], M_{1}\right)\right)$. The first relation implies that $M \cap M_{2}$ $\neq \varnothing$ and hence $M_{1} \cap M_{2} \neq \varnothing$. This contradiction shows that $h \phi\left[M_{1}\right]$ $\notin X$ and must therefore be in $Y$. Since $h$ is a homeomorphism, $\left(\phi\left[M_{1}\right], h \phi\left[M_{1}\right]\right)$ is an accumulation point of $\left\{\left(x_{i}, h\left(x_{i}\right)\right)\right\}=\left\{\left(x_{i}, y_{i}\right)\right\}$ in $X \times Y-\Delta$.

$X \times Y$ is an $M$-space. The real-valued function $f$ on $X \times Y$ defined by

$$
\begin{aligned}
f(x, y)=n \quad \text { if }(x, y)=(n, n), \\
=0 \quad \text { if }(x, y) \notin \Delta,
\end{aligned}
$$


is continuous (since $\Delta$ is both open and closed in $X \times Y$ ), closed, and $f^{-1}(r)$ is countably compact for each real $r$.

\section{REFERENCES}

1. Z. Frolik, Generalizations of compact and Lindelöf spaces, Czechoslovak Math. J. 9 (84) (1959), 172-217. MR 21 \#3821.

2. - Sums of ultrafilters, Bull. Amer. Math. Soc. 73 (1967), 87-91. MR 34 \#3525.

3. - Homogeneity problems for extremally disconnected spaces, Comment. Math. Univ. Carolinae 8 (1967), 757-763.

4. T. Isiwata, The product of $M$-spaces need not be an $M$-space, Proc. Japan Acad. 45 (1969), 154-156. MR $39 \# 6242$.

5. K. Morita, Paracompactifications of M-spaces, Proc. Japan Acad. 46 (1970), $511-513$.

6. - Topological completions and $M$-spaces (preprint).

7. J. Novák, On the Cartesian product of two compact spaces, Fund. Math. 40 (1953), 106-112. MR 15, 640.

8. W. Rudin, Homogeneity problems in the theory of Čech compactifications, Duke Math. J. 23 (1956), 409-419; correction, ibid., 633. MR 18, 324.

University of Alberta, Edmonton, Alberta, Canada 